\title{
Wavelet-Based Homomorphic De-Speckling Technique With Directional Weighted-Averaging Stage
}

\author{
Jassim M. Abdul-Jabbar \\ Computer Engineering Department, University of Basrah
}

\begin{abstract}
In this paper, a new homomorphic wavelet-based de-speckling technique is proposed. The technique combines a spatial domain processing with another transform domain processing in a homomorphic environment. The spatial domain nonlinear processing is based on replacing each log-transformed pixel value of the speckled image by a weighted-average of the logtransformed pixel values in the neighborhood of that pixel. The weighted averaged logtransformed image is then subjected to wavelet thresholding for further nonlinear transform domain processing. An exponential operation on the filtered output is used to simulate the final homomorphic antilog-transformation stage and to obtain the de-speckled image. Quantitative and qualitative measures prove the superiority of the proposed technique for the applications of image classification and recognition in ultrasonic and SAR systems.
\end{abstract}

Keywords: Speckle noise, homomorphic environment, weighted-average, wavelet thresholding

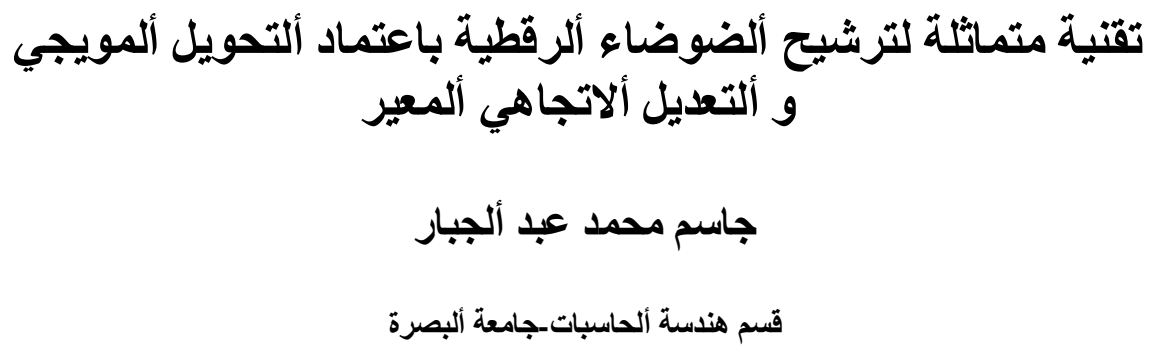

الخلاصة

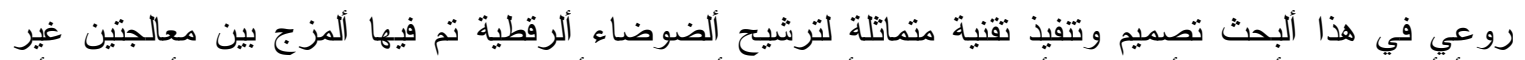

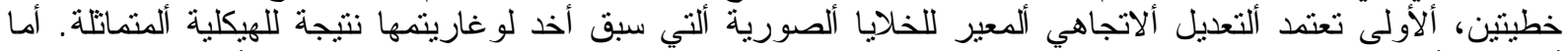

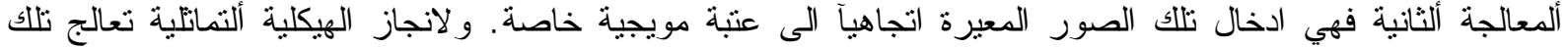
ألصور أسياً للحصول على ألصورة ألمرشحة. ألقياسات ألكمية و ألنوعية أثنتت تفوق أداء ألثقنية ألمقترحة وملائمنها لتطبيقات ألتصنيف و ألثمييز في أنظمة ألسونار و ألر ادار . النيات 


\begin{tabular}{llll} 
Al-Rafidain Engineering & Vol.17 & No.5 & October 2009 \\
\hline
\end{tabular}

\section{Introduction}

Speckle noise occurs in all types of imagery acquired from coherent imaging systems, such as laser, ultrasound and SAR imagery. Speckle is caused by random constructive and destructive interference of de-phased but coherent return waves reacted by the many elementary scatterers contained within each imaged resolution cell [1]-[3]. Speckle affects all coherent imaging systems and thus regarded as multiplicative noise in the speckled image [2],[4]. A de-speckling technique is usually required to remove this noise from such image while retaining as much as possible its important features .

The adaptive image restoration techniques ( such as Lee filter, Frost filter, and Kuan filter [3],[5]) belong to post-image formation filters and the filtering process is based on image statistical information. These standard adaptive de-speckling filters yield easily computable equations, but do not preserve fine details in complex images. Homomorphic transformation can sometimes be a reasonable way of converting signal-dependent or pure multiplicative noise to an additive noise, which then can be filtered appropriately. The homomorphic approach is initially introduced by Jain [6], where a speckled image is first log-transformed to make the multiplicative speckle noise additive. The log-transformed image is then subjected to Wiener filtering, followed by an exponential operation on the filtered output to obtain the de-speckled image (see Fig. 1). However, this method essentially, being low-pass filtering blurs many important signal features.

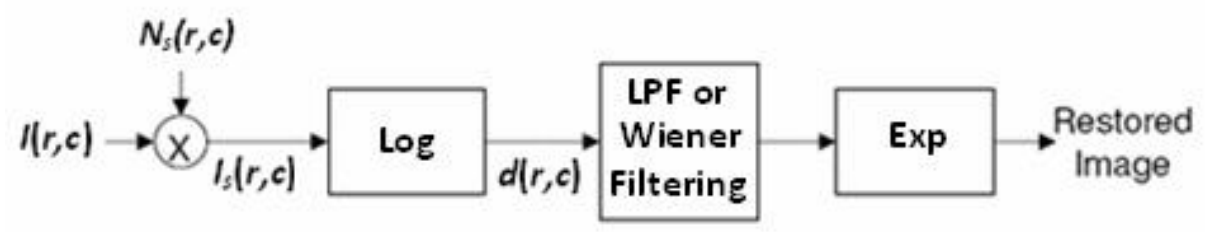

Fig. 1 A signal flow diagram of a typical homomorphic transformation.

Recently, the multiscale wavelet transform has been used with considerable success for recovering signal from noisy data [5],[7],[8]. However, by employing wavelet shrinkage based on a Bayesian formalism, it is possible to recover signals from the noisy data more effectively than by using the thresholding techniques[9]. It should be noted that, in Bayesian estimators, the wavelet coefficients are assumed to be mutually independent[8]. In practice, these coefficients show dependency within the scale as well as across the scales [7]. The drawback of this method is also its high computational complexity.

More recently, there has been much research on many new wavelet-based techniques for speckle reduction in SAR images [3],[4],[10]-[12]. It has been shown in [3] that wavelet thresholding (instead of Wiener filtering) in a homomorphic framework can provide a better reduction of the speckle noise (see Fig. 2).Theoretically, it is known that the multiplicative noise is the ratio of the standard deviation to the signal mean value [13]. Such ratio is usually treated as a constant at every pixel in the SAR image. Based on this assumption, which is not the case in practice, most of the filters for speckle reduction are designed. In this paper, another homomorphic wavelet-based de-speckling technique is proposed. The technique combines two processing domains; the spatial domain and the transform domain in a homomorphic 
Abdul - Jabbar : Wavelet-Based Homomorphic De-Speckling Technique With......

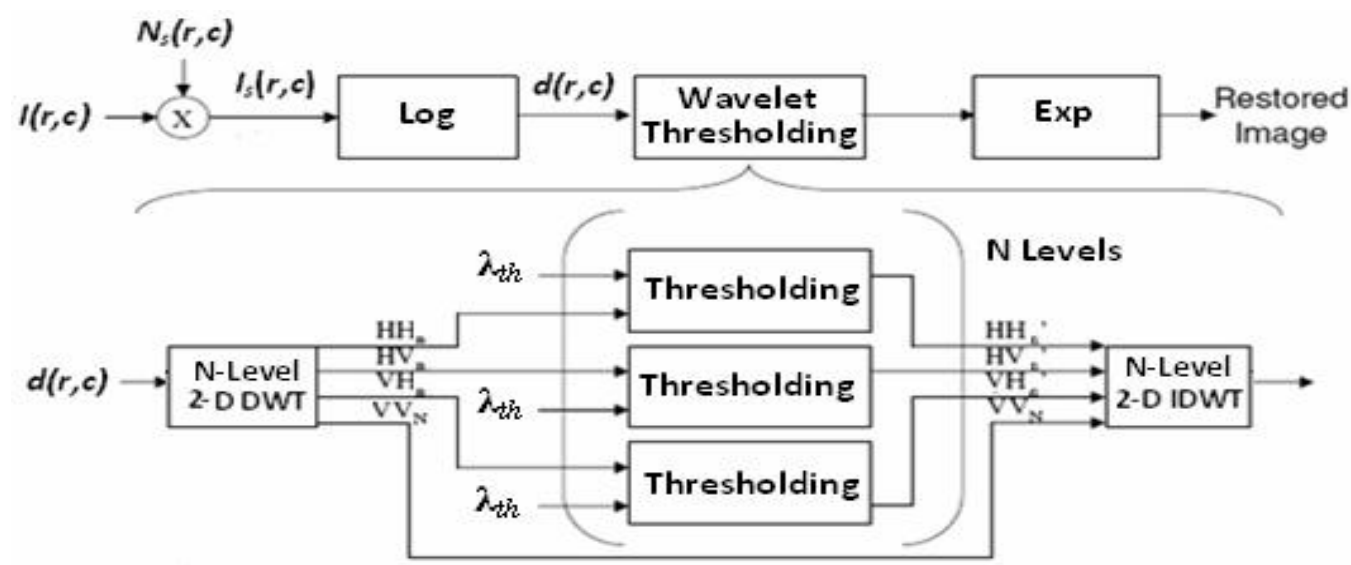

Fig. 2 A signal flow diagram of a wavelet thresholding in a homomorphic transformation

environment. The spatial domain nonlinear processing is based on replacing each logtransformed pixel value of the speckled image by a directional weighted-average of the logtransformed pixel values in the neighborhood of that pixel. The weighted-averaged logtransformed image is then subjected to wavelet thresholding for further nonlinear transform domain processing. An exponential operation on the filtered output is used to simulate the final homomorphic antilog-transformation stage and to obtain the de-speckled image. Speckle model is outlined in Section II. Section III describes the proposed homomorphic wavelet-based technique. Different quality measures are described in Section IV. Performance evaluation and a comparative study is illustrated in Section V. Finally, conclusions are given in Section VI.

\section{Speckle Model}

To explain the mechanism of speckle noise in ultrasound and SAR images, an essential multiplicative/additive compound noise process model is formulized as

$$
I_{s}(r, c)=I(r, c)+I(r, c) \times S(r, c)
$$

where $I(r, c)$ represents the true values of the original image pixels, $S(r, c)$ denotes the speckle noise pixels introduced into the image to produce the degraded (speckled) image pixels $I_{s}(r, c)$, and " $r, c$ " represents the pixel location. The model in eq. (1) can then be reduced to the following multiplicative form:

$$
I_{s}(r, c)=I(r, c) \times N_{s}(r, c)
$$

where

$$
N_{\mathrm{s}}(r, c)=1+S(r, c)
$$

Applying homomorphic transformation, the log-transform of eq. (2) yields an additive speckle model of the type given by

$$
\begin{gathered}
d(r, c)=f(r, c)+N(r, c) \\
\text { where } d(r, c)=\log \left\{I_{s}(r, c)\right\}, \\
f(r, c)=\log \{I(r, c)\},
\end{gathered}
$$




\section{$\begin{array}{llll}\text { Al-Rafidain Engineering } & \text { Vol.17 } & \text { No.5 } & \text { October } 2009\end{array}$}

and $\quad N(r, c)=\log \left\{N_{s}(r, c)\right\}$

Since the original image detected pixel values can be factorized into two components as follows:

$$
I(r, c)=L(r, c) \times R(r, c)
$$

where $L(r, c)$ is the luminance and $R(r, c)$ is the reflectance of the scene, then eq. (5b) can be rewritten as

$$
f(r, c)=L L(r, c)+L R(r, c)
$$

where $\quad L L(r, c)=\log \{L(r, c)\}$

and $\quad L R(r, c)=\log \{R(r, c)\}$

Thus, eq. (4) can then be rewritten as

$$
d(r, c)=L L(r, c)+L R(r, c)+N(r, c)
$$

which means that each log-transformed pixel in the speckled image $d(r, c)$ consists three additive components; A low frequency one $L L(r, c)$ and two high frequency components $L R(r, c)$ and $N(r, c)$. However, applying any low pass filtering ( LPF ) as in Fig. 1 on the log-transformed speckled image pixel $d(r, c)$ can isolate the high frequency noise component $N(r, c)$, but it will also blur many important signal features due to elimination of high frequency image pixel component $\operatorname{LR}(r, c)$. An alternative way is to use wavelet thresholding in the homomorphic framework as shown in Fig. 2 . In such a frame, the log-transformed image $d(r, c)$ is applied to a 2-D discrete wavelet transform (DWT). Then, by a proper thresholding the high frequency noise components of eq. (9), $N(r, c)$ can approximately be eliminated or even reduced, leaving the high frequency image component $L R(r, c)$ together with $L L(r, c)$ to act as inputs to a 2-D inverse discrete wavelet transform (IDWT), forming the final restored image. This technique provides better reductions in the speckle noise as compared with that of the spatial-domain filters. Many modified wavelet-based and homomorphic wavelet-based de-speckling techniques are designed as in [3],[4],[7]-[12]. Most of those techniques suffer from high computational complexity drawbacks. In the next section, a new homomorphic wavelet-based technique is proposed.

\section{Proposed Technique}

Basically, image noise filtering can be viewed as replacing each pixel value in the noisy image with a new value depending on the local context. Such filtering process should ideally vary from pixel to pixel based also on the local context [14]. This goal can be achieved either in the image domain (spatial domain processing) or in the frequency domain (transform domain processing). The proposed technique combines both image and transform nonlinear processes in a homomorphic environment. A signal flow diagram of the proposed homomorphic de-speckling technique is shown in Fig. 3. The speckled image pixels $I_{s}(r, c)$ is initially log-transformed to $d(r, c)$ which is an image with additive noise as given in eq. (4). The two nonlinear processing stages are described as follows: 


\section{Abdul - Jabbar : Wavelet-Based Homomorphic De-Speckling Technique With......}

\section{A. Directional Weighted-Averaging Stage}

The image domain nonlinear processing is based on replacing each log-transformed pixel value $d(r, c)$ by a directional weighted-average of the log-transformed pixel values in the neighborhood of that pixel. In such process, eight directional differences $d_{1}, d_{2}, \ldots d_{8}$ of each $3 \mathrm{X} 3$ window centered at each log- transformed pixel value $d(r, c)$, as shown in Fig. 4, are calculated as

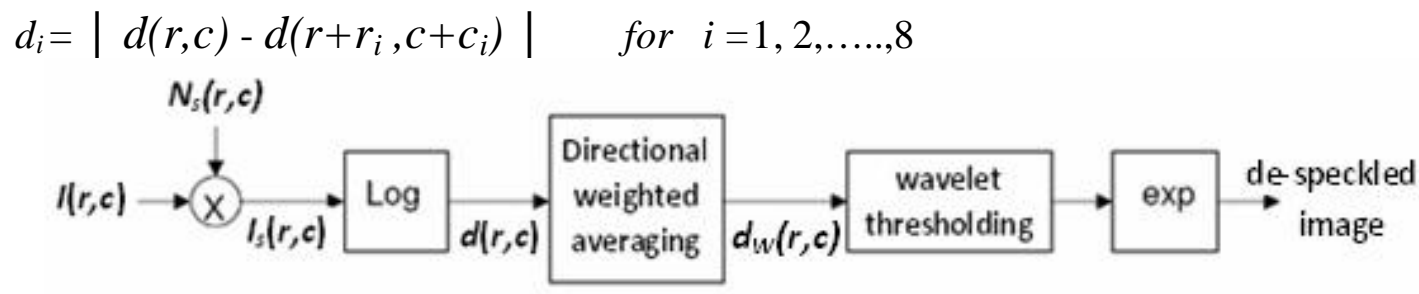

Fig. 3 A signal flow diagram of the proposed homomorphic de-speckling technique.

Where :

$r_{1}=r_{5}=c_{3}=c_{7}=0$, and $r_{2}=r_{3}=r_{4}=c_{1}=c_{2}=c_{8}=-r_{6}=-r_{7}=-r_{8}=-c_{4}=-c_{5}=-c_{6}=1$.

The choice of normalized directional weights $W_{1}, W_{2}, \ldots . W_{8}$, corresponding to the $3 \times 3$ window, is based on the differences $d_{1}, d_{2}, \ldots d_{8}$, respectively. These normalized weights take the values in the range $[0,1]$ by applying the following criterion: a small weight $W_{i}$ must be applied to the pixel in the neighborhood with the direction of large difference $d_{i}$ to reduce its contribution in the averaging process [14]. Thus, the following relations are used:

$$
W_{i}=\left(W d_{i} / W_{\max }\right) \quad \text { for } i=1,2, \ldots \ldots, 8
$$

where

$$
W d_{i}=\left[1 /\left(\alpha+d_{i}\right)\right]^{\beta} \quad \text { for } i=1,2, \ldots, 8
$$

and

$$
W_{\max }=\max \left\{W d_{1}, W d_{2}, \ldots, W d_{8}\right\}
$$

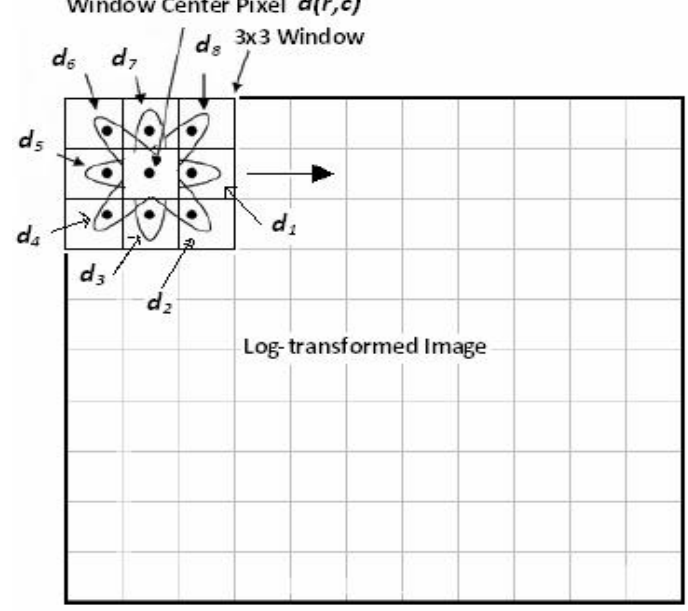

Fig. 4 Directional weighted-averaging of log-transformed center pixel of a $3 \times 3$ window in a logtransformed image based on directional differences $d_{1}, d_{2}, \ldots, d_{8}$. 
with $\alpha$ and $\beta$ as very small constants. A directional weighted-averaged pixel $\tilde{d}(r, c)$ can be calculated as

$$
\hat{d}(r, c)=\left(1 / W_{T}\right) \sum_{i=0}^{8} w_{i} d\left(r+r_{i}, c+c_{i}\right)
$$

where

$$
W_{T}=\sum_{i=0}^{8} W_{i}
$$

with $W_{0}=1$, and $r_{o}=c_{o}=0$.

To get a better performance in the output of the directional weighted-averaging stage $d_{W}(r, c)$, the central pixel $d(r, c)$ in the $3 \mathrm{X} 3$ window is either replaced by the $\hat{d}(r, c)$ value or returns its original value, depending on the followings:

$$
d_{W}(r, c)=\left\{\begin{array}{lll}
\hat{d}(r, c) & \text { for } & \operatorname{dif}(r, c)>T_{t h} \\
d(r, c) & \text { for } & \operatorname{dif}(r, c) \leq T_{t h}
\end{array}\right\}
$$

where $\operatorname{dif}(r, c)$ is an element, in a difference matrix $D$ which has the same size of original image with the corresponding image matrix locations $(\mathrm{r}, \mathrm{c})$. $\operatorname{dif}(r, c)$ is calculated as follows:

$$
\operatorname{dif}(r, c)=|d(r, c)-\hat{d}(r, c)|
$$

and

$$
T_{t h}=0.5 \delta\left[d i f_{\max }+d i f_{\min }\right]
$$

where

$$
\begin{aligned}
& d i f_{\text {max }}=\max \{D\}, \\
& d i f_{\text {min }}=\min \{D\},
\end{aligned}
$$

and $\delta$ is a factor chosen for best performance.

\section{B. Wavelet Thresholding}

As shown in Fig. 3, the resulting weighted-averaged image $d_{W}(r, c)$ is further subjected to a wavelet thresholding for another nonlinear transform domain processing. A 2-level 2-D DWT is applied with a specified thresholding level on all details. The threshold level $\lambda_{t h}$ is chosen to be

$$
\lambda_{\text {th }}=\mu \sigma_{n}
$$

where $\sigma_{n}$ is the noise standard deviation of the weighted-averaged image which can be estimated as given by Donoho [15]

$$
\sigma_{n}=\text { median }\left\{\left|H H_{\text {subband }}\right|\right\} / 0.6745
$$

and $\mu$ is a multiplicative constant chosen for best performance. $H H_{\text {subband }}$ is the diagonal detail coefficient of the first decomposition level.

After thresholding, different wavelet coefficients are fed to 2-level 2-D IDWT. As a final stage, an exponential operation on the reconstructed output of the 2-D IDWT is used to simulate the final homomorphic antilog-transformation stage and to obtain the de-speckled image. Since the mean of the log-transformed speckle noise is biased [4], an adjustment is needed to remove the associated mean bias to avoid extra distortion in the restored image, especially for high noise levels. This adjustment is provided for in the final stage. 
Abdul - Jabbar : Wavelet-Based Homomorphic De-Speckling Technique With......

\section{Iv. Objective Assessment Parameters}

Different objective assessment parameters are used to evaluate the performance of the proposed de-speckling technique. These parameters are Noise Mean Value (NMV), Noise Variance (NV), Noise Standard Deviation (NSD), Mean Square Difference (MSD), Equivalent Number of Looks (ENL), Deflection Ratio (DR), and Pratt's figure of Merit (FOM) [4],[5],[10][12].

$\mathrm{NV}$ determines the contents of the speckle in the image. A lower variance gives a "smoother and cleaner" image as more speckle is removed, although, it not necessarily depends on the intensity. The formulas for calculating NMV, NV and NSD are given by

$$
\begin{aligned}
& \mathrm{NMV}=\frac{\sum_{r, c} I_{d}(r, c)}{R * C}, \\
& \mathrm{NV}=\frac{\sum_{r, c}\left[I_{d}(r, c)-\mathrm{NMV}\right]^{2}}{R * C},
\end{aligned}
$$

and

$$
\mathrm{NSD}=\sqrt{\mathrm{NV}}
$$

where $I_{d}(r, c)$ represents the de-speckled image pixels and $R^{*} C$ is image size.

MSD indicates average difference of the pixels throughout the image. It can be calculated as

$$
\operatorname{MSD}=\frac{\sum_{r, c}\left[I_{S}(r, c)-I_{d}(r, c)\right]^{2}}{R * C}
$$

A lower MSD indicates a smaller difference between the original and de-speckled image. This means that there is a significant filter performance. Nevertheless, it is necessary to be very careful with the edges [11], [12]. ENL is another good approach of estimating the speckle noise level in an image over uniform regions. A simple formula for ENL is given by

$$
\mathrm{ENL}=\frac{\mathrm{NMV}^{2}}{\mathrm{NSD}^{2}}
$$

Larger value of ENL usually corresponds to a better quantitative performance. The value of ENL also depends on the size of the tested region. Theoretically, a larger region will produces a higher ENL value than over a smaller region but it also trade-off the accuracy of the readings. Due to the difficulty in identifying uniform areas in the image, the image is divided into smaller areas of $25 \times 25$ pixels. The ENL for each of these smaller areas is obtained and finally the average of these ENL values is calculated. Unfortunately, the ENL carries no information on the resolution degradation and because of that, it is often used jointly with other parameter like the Signal-to-Mean-Square-Error Ratio (S/MSE) or MSD [4]. ENL, in our experiments is jointly obtained with MSD. The significance of obtaining both MSD and ENL measurements is to analyze the performance of the de-speckling techniques on the overall region as well as in smaller uniform regions. DR is also used here as a performance estimator. A proposed formula for this deflection is given by [4],[11]

$$
\mathrm{DR}=\frac{1}{R * C} \sum_{r, C}\left(\frac{I_{d}(r, c)-\mathrm{NMV}}{\mathrm{NSD}}\right)
$$




\section{$\begin{array}{llll}\text { Al-Rafidain Engineering } & \text { Vol.17 } & \text { No.5 } & \text { October } 2009\end{array}$}

The ratio DR should be higher at pixels with stronger reflector points and lower elsewhere. To compare edge preservation performances of different speckle reduction schemes, the Pratt's figure of merit (FOM) is adopted. It is defined as [11],[12]

$$
\mathrm{FOM}=\frac{1}{\operatorname{Max}\left\{N_{d}, N_{i}\right\}} \sum_{i=1}^{N_{d}} \frac{1}{1+m E_{d}^{2}}
$$

where $N_{d}$ and $N_{i}$ are the number of detected and ideal edge pixels, respectively, $E_{d}$ is the Euclidean distance between the $i$ th detected edge pixel and the nearest ideal edge pixel, and $m$ is a constant typically set to $1 / 9$. FOM takes values in the range $[0,1]$ with unity for ideal edge detection.

\section{Performance Evaluation And Comparison}

The effectiveness of the proposed technique is demonstrated by comparing its objective and subjective performances with four classical de-speckling techniques (such as VisuShrink (HardThresholding), BayesShrink, NormalShrink, and SUREShrink [5],[10]).The comparison also includes three recent techniques; KalmanShrink [11], SmoothShrink [4], and POSAShrink [12]. ERS SAR Precision Image (PRI) standard of Buenos Aires area is used as an original noisy image (see Fig. 5). Such image is from remote sensing satellite ERS-2, with 8-bit, 242x242 pixels. The de-speckled images, processed by using classical and recent techniques, including the proposed one are also shown in Fig. 5. For best performance of the proposed techniques, the processing constants and the parameters are chosen as $\alpha=0.001, \beta=0.1, \delta=0.0123$, and $\mu=1.25$. From Fig. 5, it can be seen that the proposed technique is a successful tool for eliminating speckle without distorting useful image information ,i.e., keeping important image edges preserved. It should be noted that the proposed technique is accomplished with acceptable computational complexity.

Table 1 shows the objective assessment parameters for different images shown in Fig. 5. The quantitative results of Table 1 again highlight the ability of the proposed technique to eliminate speckle, preserving the useful image information, since it has a good NMV preservation and gives the best variance reduction $(\mathrm{NSD}=26.2757)$. Since a successful despeckling technique will not significantly affect the mean intensity within a homogeneous region, the proposed one acts well in that sense too. As illustrated in Table 1, the proposed technique also outperforms the others in terms of ENL. Larger ENL value usually corresponds to a better quantitative performance. From edge preservation point of view, the DR and FOM values indicate the superiority of the proposed technique.

\section{Vi. Conclutions}

A new homomorphic wavelet-based de-speckling technique for SAR and ultrasound images has been proposed with directional weighted-averaging stage. The homomorphic environment converts multiplicative speckle model into an additive noise one. In such environment, the proposed technique combines two nonlinear processes; the image domain processing and the transform domain processing. The image domain nonlinear processing is based on replacing each log-transformed pixel value of the speckled image by a directional weighted-average of the logtransformed pixel values in the neighborhood of that pixel. The weighted-averaged logtransformed image is then subjected to wavelet thresholding for further nonlinear transform 
Abdul - Jabbar : Wavelet-Based Homomorphic De-Speckling Technique With......

domain processing. As a final homomorphic stage, antilog-transformation is used to obtain the de-speckled image. This technique is compared with other classical and recent techniques. Subjectively, a cleaner de-speckled image is obtained which indicates the potential improvement in the detection performance of the proposed technique. This refers to the excellent image visual quality in such technique.

As illustrated in Table 1, the objective assessment parameters of such technique highlight its superiority. Good NMV preservation and best variance reduction (lowest NSD value) show the ability to eliminate speckle, preserving the useful image information. A better quantitative performance is also expected as ENL value is the highest. The considerable increase in DR ratio as well as FOM value, strongly indicate the improvement in detection performance. Besides, the method is computationally efficient and can significantly reduce the speckle while preserving the resolution of the original image, only with 2-level wavelet decomposition avoiding blocky effect.

The effectiveness of the proposed technique encourages the possibility of using the approach in a number of SAR and ultrasonic applications such as classification and recognition. Finally, it should be noted that all implementations are executed by MATLAB on a P4 PC.

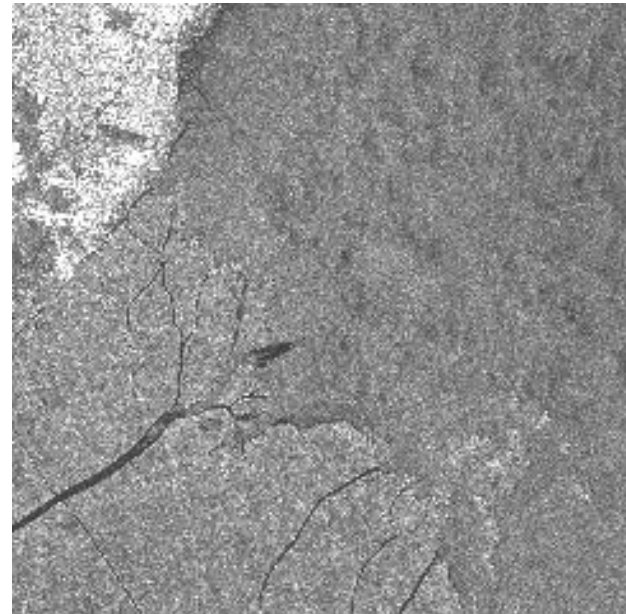

(a) Original

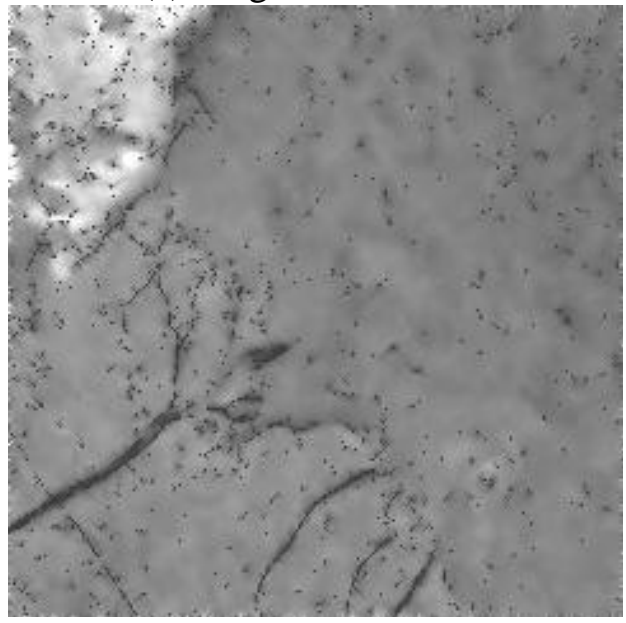

(c) NormalShrink

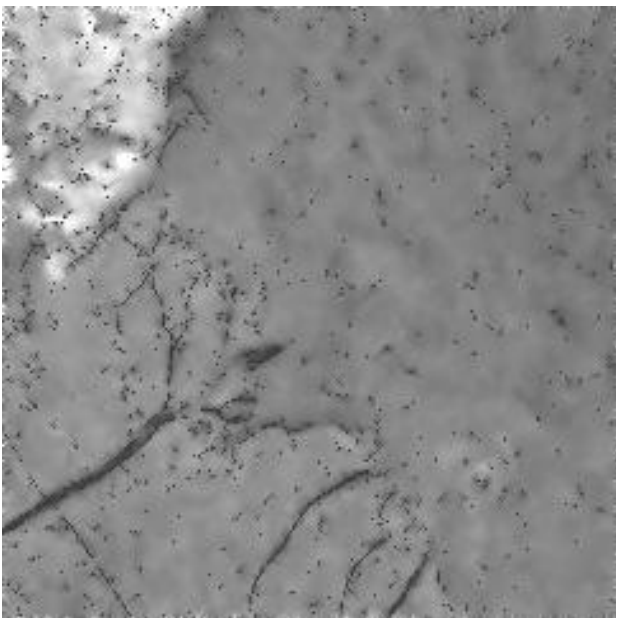

(b) VisuShrink

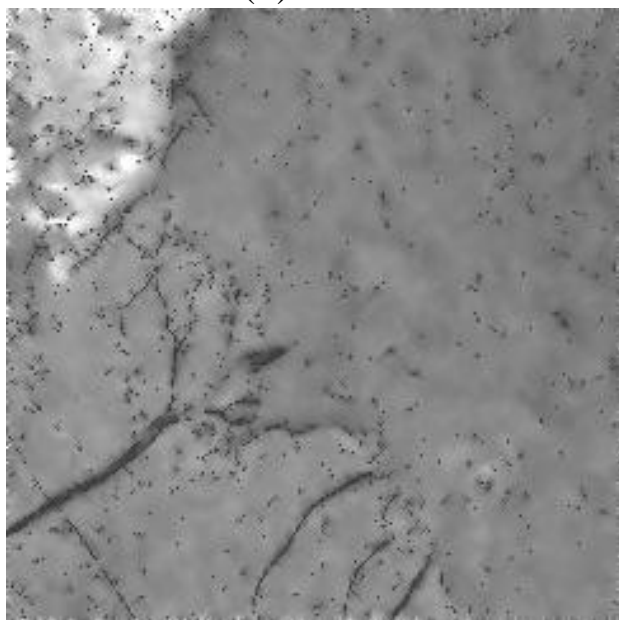

(d) BayesShrink

Fig. 5 Original SAR speckled image and restored images of different techniques. 
$\begin{array}{llll}\text { Al-Rafidain Engineering } & \text { Vol.17 } & \text { No.5 } & \text { October } 2009\end{array}$

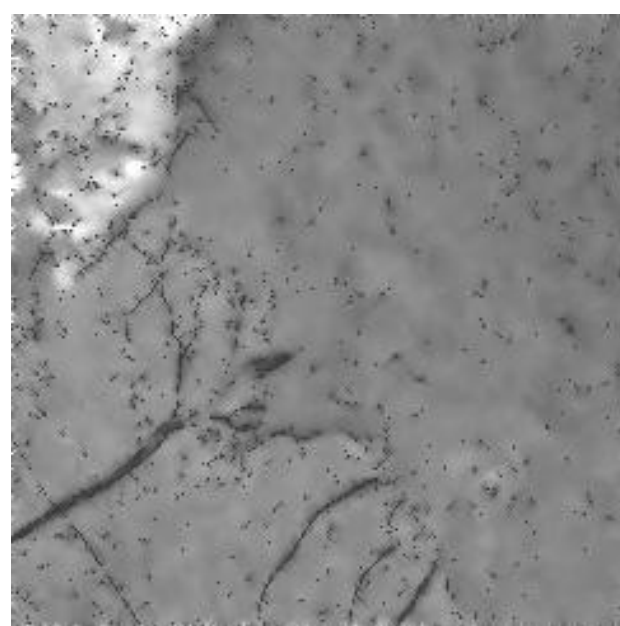

(e) SUREShrink

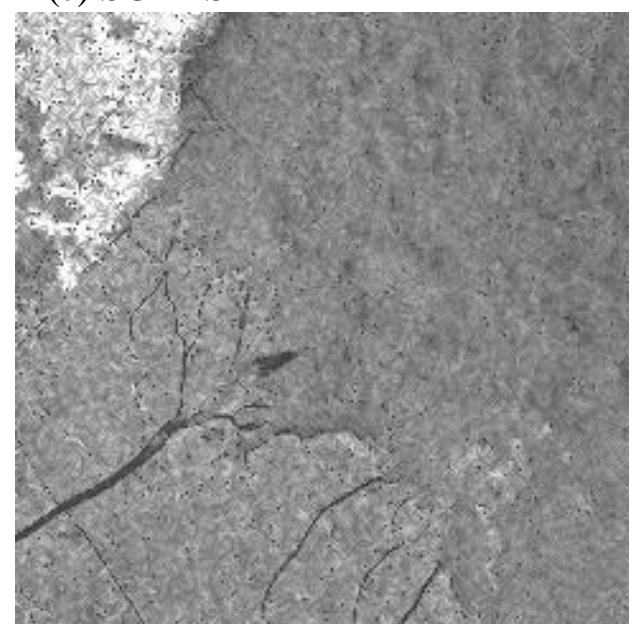

(g) SmoothShrink

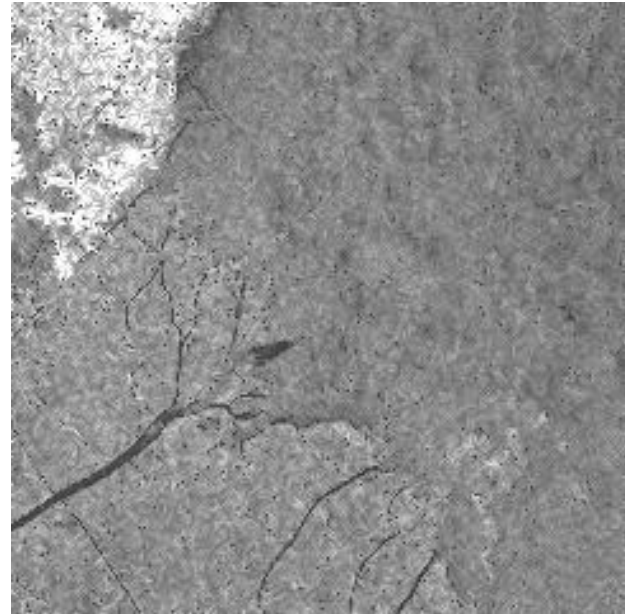

(f) KalmanShrink

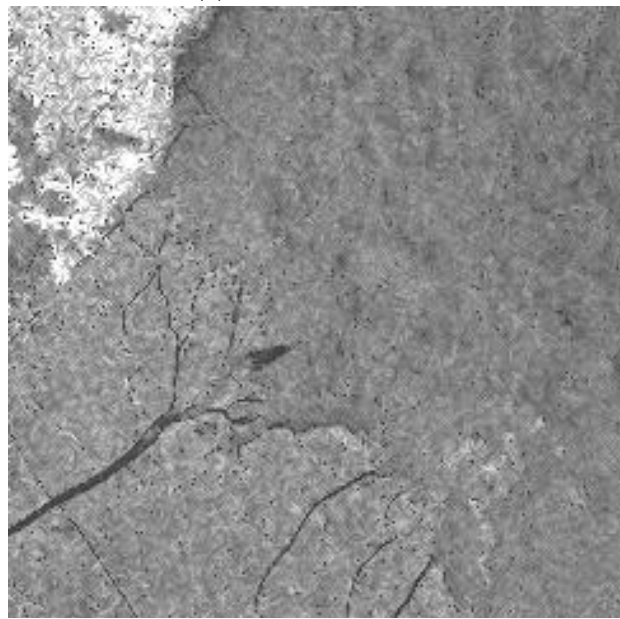

(h) POSAShrink

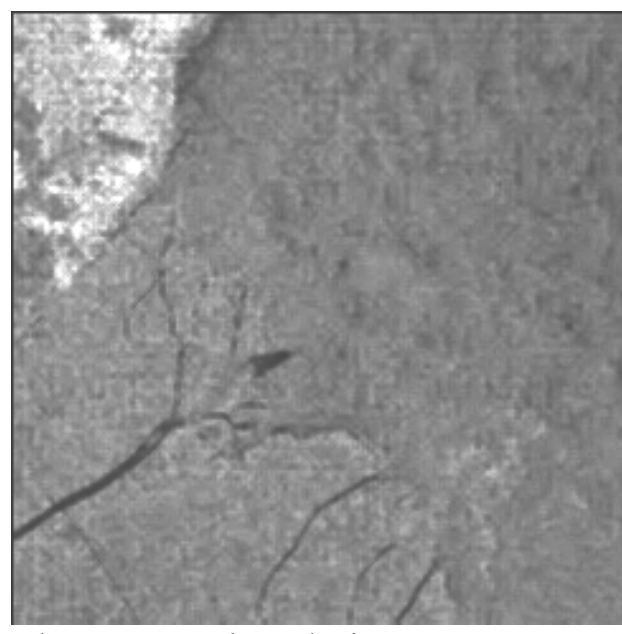

(i) The Proposed Technique

Fig. 5 (continued) 
Abdul - Jabbar : Wavelet-Based Homomorphic De-Speckling Technique With......

Table 1: Objective assessment parameters for different de-speckling techniques

\begin{tabular}{|c|c|c|c|c|c|c|}
\hline \multirow{2}{*}{ Techniques } & \multicolumn{7}{|c|}{ Objective assessment parameters } \\
\cline { 2 - 7 } & MSD & NMV & NSD & ENL & DR & FOM \\
\hline Original noisy image & - & 90.0890 & 43.9961 & 11.0934 & $2.5580 \mathrm{e}-017$ & 0.3027 \\
\hline VisuShrink (HT) & 855.3030 & 88.4311 & 32.8688 & 39.0884 & $7.8610 \mathrm{e}-016$ & 0.4519 \\
\hline NormalShrink & 732.2345 & 88.5233 & 33.3124 & 36.8464 & $6.7354 \mathrm{e}-016$ & 0.4576 \\
\hline BayesShrink & 724.0867 & 88.9992 & 36.8230 & 36.0987 & $1.0534 \mathrm{e}-015$ & 0.4581 \\
\hline SureShrink & 716.6344 & 87.9920 & 32.8978 & 38.3025 & $2.4005 \mathrm{e}-015$ & 0.4520 \\
\hline KalmanShrink & 867.1277 & 90.0890 & 32.6884 & 39.0884 & $3.2675 \mathrm{e}-015$ & 0.4591 \\
\hline SmoothShrink & 867.1277 & 90.0890 & 32.6884 & 39.0884 & $3.2675 \mathrm{e}-015$ & 0.4591 \\
\hline POSAShrink & 867.1277 & 90.0890 & 32.6884 & 39.0884 & $3.2675 \mathrm{e}-015$ & 0.4591 \\
\hline Proposed technique & 773.2842 & 88.8417 & 26.2757 & 45.7284 & $3.6636 \mathrm{e}-015$ & 0.4652 \\
\hline
\end{tabular}

\section{References}

[1] J. Xiao, J. Li, P. R. China, and A. Moody," A detail-preserving and flexible adaptive filter for speckle suppression in SAR imagery", Int. J. Remote Sensing, Vol. 24, No. 12, pp. 2451$2465,2003$.

[2] Z. Long, and N. H. Younana,"Denoising of images with multiplicative noise corruption", Department of Electrical \& Computer Engineering and Diagnostic Instrumentation \& Analysis Laboratory, Mississippi State University, 2005.

www.eurasip.org/Proceedings/Eusipco/Eusipco2005/defevent/papers/cr1755.pdf

[3] E. Hui-On Ng," Speckle noise reduction via homomorphic elliptical threshold rotations in the complex wavelet domain", M.Sc. thesis, Electrical and Computer Engineering in the University of Waterloo, Waterloo, Ontario, Canada, 2005.

[4] M. Mastriani, A. E. Giraldez," Smoothing of coefficients in wavelet domain for speckle reduction in Synthetic Aperture Radar images", The International Congress for Global Science and Technology (ICGST), International Journal on Graphics, Vision and Image Processing (GVIP), GVIP Special Issue on Denoising, pp.1-8, 2007. www.icgst.com

[5] L. Gagnon and A. Jouan," Speckle filtering of SAR images - A comparative study between complex-wavelet-based and standard filters" Proc. SPIE, Vol. 3169, pp. 80-91, 1997.

[6] A. K. Jain, Fundamentals of Digital Image Processing. Englewood Cliffs, NJ: Prentice-Hall Ltd., 1989.

[7] R. W. Buccigrosi and E. P. Simoncelli, "Image compression via joint statistical characterization in the wavelet domain," IEEE Trans. Image Process., Vol. 8, No. 12, pp. 16881701, Dec. 1999.

[8] A. Antoniadis, J. Bigot, and T. Sapatinas, "Wavelet estimators in nonparametric regression: a comparative simulation study," J. Stat. Softw., Vol. 6, pp. 1-83, 2001.

[9] A. Achim, P. Tsakalides, and A. Bezarianos, "SAR image denoising via Bayesian wavelet shrinkage based on heavy-tailed modeling," IEEE Trans. Geosci. Remote Sens., Vol. 41, No. 8, pp. 1773-1784, Aug. 2003.

[10] M. Mastriani and A. E. Giraldez," Enhanced directional smoothing algorithm for edgepreserving smoothing of synthetic-aperture radar images", Measurement Science Review, Vol. 4, Sec. 3, pp. 1-11, 2004. 


\section{Al-Rafidain Engineering $\quad$ Vol.17 $\quad$ No.5 $\quad$ October 2009}

[11] M. Mastriani and A. E. Giraldez," Kalman's Shrinkage for Wavelet-Based De-speckling of SAR Images", International Journal Of Intelligent Technology, Vol. 1, No. 3, pp. 190-196, 2006. [12] M. Mastriani," New Wavelet-Based Superresolution Algorithm for Speckle Reduction in SAR Images", International Journal of Computer Science, Vol.1 No. 4, pp. 291-298, 2007.

[13] B. Matalona, M. Elad, and M. Zibulevskya," Improved denoising of images using modelling of a redundant contourlet transform", EE Department and CS Department, The Technion, Haifa 32000, Israel. www.cs.technion.ac.il/ elad/publications/conferences/2005/28 SPIE DenoisingCT.pdf

[14] H. V. Nejad, H. R. Pourreza, and H. Ebrahimi," A novel fuzzy technique for image noise reduction," Proc. of World Academy of Science, Engineering and Technology, Vol.14, pp. 390395, August 2006.

[15] D. L. Donoho, "Denoising by softthresholding," IEEE Trans. Inf. Theory, Vol. 41, No. 3, pp. 613-627, May 1995.

The work was carried out at the college of Engg. University of Mosul 